\title{
Une stratégie multi-échelle pour l'étude paramétrique de détails géométriques au sein de structures en contacts multiples
}

\author{
Pierre-Alain Boucard — Sandrine Buytet \\ Pierre-Alain Guidault \\ LMT-Cachan (ENS de Cachan/CNRS/Université Paris 6/PRES UniverSud Paris) \\ 61 avenue du Président Wilson, F-94230 Cachan cedex \\ \{boucard, buytet, guidault\}@lmt.ens-cachan.fr
}

\begin{abstract}
RÉSUMÉ. Dans ce papier nous présentons une méthode multi-échelle dédiée à l'étude paramétrique de détails géométriques dans une structure en contacts multiples avec frottement. Le premier point de la stratégie utilise une technique de résolution micro-macro. Elle est basée sur une décomposition du domaine en sous-structures et interfaces, permettant de résoudre indépendamment les problèmes " micro " dans les différentes sous-structures, et de transmettre l'information "macro" via les interfaces. Ensuite, une stratégie multirésolution est introduite pour réduire les temps de calcul lorsque les paramètres varient. Finalement, le dernier point permet de décrire la géométrie du détail indépendamment du maillage. Il combine une méthode d'enrichissement locale, la X-FEM et l'utilisation de fonctions level set permettant «d'activer » facilement le détail.

ABSTRACT. This paper presents a multiscale strategy dedicated to the parametric study of structural details inside a structure with multiple friction contacts. The first point of the method uses a micro-macro approach. It is based on a domain decomposition in substructures and interfaces, which involves the resolution of independent "micro" problems in each substructures and transfers the "macro" information only via the interfaces. In a second point, a multiresolution strategy is used in order to reduce the computational cost in the case of problems with design variables. Moreover, the last one consists in modelling the geometry of details without remeshing. On the one hand, it uses a local enrichissement method, the X-FEM, and, on the other hand, level set functions enable one to easily "activate" the detail.

MOTS-CLÉS : stratégie multi-échelle, multirésolution, contact avec frottement, X-FEM.

KEYWORDS: multiscale strategy, multiresolution, frictional contact, X-FEM.
\end{abstract}

DOI:10.3166/REMN.16.1011-1036 @ 2007 Lavoisier, Paris 


\section{Introduction}

L'étude paramétrique est au cœur d'un grand nombre de problèmes de calcul de structures. Elle étudie la sensibilité de la réponse de la structure par rapport à certains aléas appelés paramètres de conception. La construction de surfaces de réponse lorsque ces paramètres prennent différentes valeurs dans des intervalles a priori connus, permet de déterminer rapidement la gamme raisonnable (voire optimale) de conception ou d'utilisation. Les études paramétriques concernent généralement les propriétés mécaniques du matériau (module d'Young ou coefficient de Poisson), les conditions aux limites, les conditions de contact (jeu, coefficient de frottement) mais peuvent également porter sur la géométrie de la structure ou sur la prise en compte de détails structuraux (positions, formes). Notons que la frontière entre étude paramétrique et optimisation est très étroite et que la première constitue souvent un outil indispensable à la seconde. Dans le cadre de notre étude nous considérons deux types de variables de conception, les paramètres caractérisant des interfaces de contact et les paramètres décrivant un détail structural. L'analyse paramétrique engendrant un nombre important de calculs, il est crucial d'introduire une stratégie de résolution adaptée permettant de réduire les temps de calcul lorsque les paramètres évoluent. Différentes stratégies dites mutirésolutions sont présentées dans le cadre de l'optimisation topologique par exemple dans (Kim et al., 2000). La méthode multirésolution utilisée ici (Boucard et al., 2003; Boucard et al., 2004) se base sur la méthode LATIN développée au LMT-Cachan (Ladevèze, 1999).

Par ailleurs, la prise en compte d'un détail structural implique de considérer le phénomène muti-échelle qu'il introduit : celui-ci ayant évidemment une influence locale mais aussi, dans une moindre mesure, une influence globale. A cette fin, on considère l'approche micro-macro (Ladevèze et al., 2001) qui s'appuie sur la décomposition du domaine en sous-structures et interfaces et où l'aspect multi-échelle est introduit au niveau des interfaces. Le dernier maillon de la chaîne de résolution porte sur la définition même du détail. Sa description se fait à partir de fonction level set (Osher et al., 1988) indépendamment du maillage éléments-finis et la méthode d'enrichissement locale X-FEM permet la prise en compte de la singularité au sein de la formulation en déplacement du problème (Moës et al., 1999; Daux et al., 2000; Sukumar et al., 2000). Différentes études couplant l'enrichissement local ont été menées dans le cadre de la stratégie multi-échelle (Fish et al., 2005; Guidault et al., 2006; Combescure et al., 2007).

Le papier présente dans un premier temps le principe général de la méthode multiéchelle et met l'accent sur les différentes étapes de résolution pour des problèmes de contact avec frottement. Après avoir donné les bases théoriques de la méthode X-FEM et de la level set, la stratégie multirésolution est introduite. Nous présentons finalement les résultats des simulations numériques. Les exemples sont traités en $2 \mathrm{D}$ et les détails structuraux sont des trous circulaires. 


\section{L'approche multi-échelle}

L'approche multi-échelle proposée ici appelée approche "micro-macro" se base sur trois points fondamentaux détaillés ci-après.

\subsection{Décomposition de domaine}

Considérons une structure élastique $\Omega$ soumise, sous l'hypothèse de petites perturbations, à un chargement $\underline{F}_{d}$ sur la portion $\partial_{2} \Omega$ de sa frontière $\partial \Omega$. Sur la partie complémentaire $\partial_{1} \Omega$, le déplacement $\underline{U}_{d}$ est imposé. Le premier point de l'approche micro-macro consiste à décomposer le système mécanique en sous-structures et en interfaces (figure 1). Chacun de ces constituants correspond à une entité mécanique à part entière caractérisée par ses variables et son comportement propre. C'est une vision naturelle dans le cas où les sous-structures sont les pièces constitutives d'un assemblage et les interfaces correspondent aux connexions entre ces pièces.

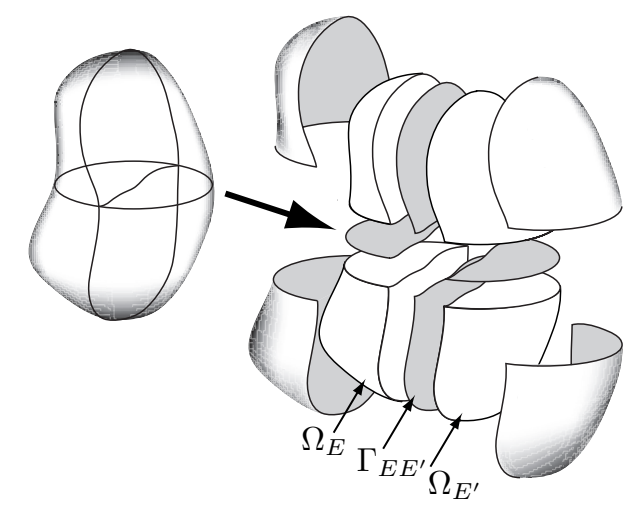

Figure 1. Décomposition du milieu en sous-structures et interfaces

Soit une sous-structure $E$, elle occupe le domaine $\Omega_{E}$, et sa frontière est notée $\partial \Omega_{E}$. Elle est soumise l'action de son environnement (les interfaces voisines) qui se traduit par une distribution d'effort $\underline{F}_{E}$ et une distribution de déplacement $\underline{W}_{E}$ (figure 2). L'interface $\Gamma_{E E^{\prime}}$ entre les deux sous-structures $\Omega_{E}$ et $\Omega_{E^{\prime}}$ traduit une relation mixte de comportement entre $\left(\underline{F}_{E}, \underline{F}_{E^{\prime}}\right)$ et $\left(\underline{W}_{E}, \underline{W}_{E^{\prime}}\right)$. L'introduction des distributions de déplacements et d'efforts d'interface confère le caractère mixte à cette méthode de décomposition de domaine. Dans la suite, on résout deux types de problèmes : un associé aux sous-structures l'autre associé aux interfaces.

Problème dans les sous-structures

Soit $\underline{u}_{E}$ le champ de déplacement de tout point de la sous-structure $\Omega_{E}, \epsilon_{E}$ et $\sigma_{E}$ sont respectivement les déformations et les contraintes correspondantes. 


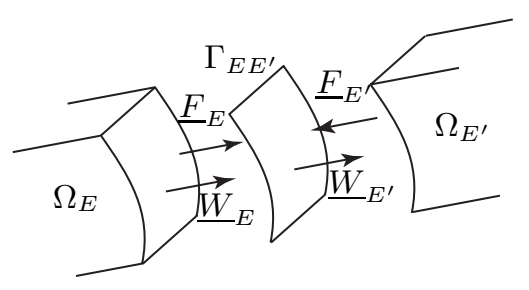

Figure 2. Echange sous-structures/interfaces

Le problème mécanique à résoudre dans chaque sous-structure consiste à déterminer $\epsilon_{E}$ et $\sigma_{E}$ verifiant :

- l'admissibilité cinématique

On cherche $\left(\epsilon_{E}, \underline{W}_{E}\right) \in \mathcal{U}_{E}$ tel que :

$$
\left\{\begin{array}{cc}
\left.\underline{u}_{E}\right|_{\partial \Omega_{E}} & =\frac{W}{E} \\
\epsilon_{E} & =\epsilon\left(\underline{u}_{E}\right)
\end{array}\right.
$$

$\mathcal{U}_{E}$ est l'espace des champs cinématiquement admissibles;

- l'équilibre au sens faible (on suppose que la répartition d'effort volumique est nulle).

On cherche $\left(\sigma_{E}, \underline{F}_{E}\right) \in \mathcal{F}_{E}$ tel que :

$$
\forall\left(\epsilon_{E}^{*}, \underline{W}_{E}^{*}\right) \in \mathcal{U}_{E}^{*}, \quad \int_{\Omega_{E}} \sigma_{E}: \epsilon_{E}^{*} d \Omega-\int_{\partial \Omega_{E}} \underline{F}_{E} \cdot \underline{W}_{E}^{*} d \Gamma=0
$$

$\mathcal{F}_{E}$ est l'espace des champs statiquement admissibles;

- la loi de comportement (on suppose que le comportement est élastique linéaire).

$$
\sigma_{E}=\mathbf{D} \epsilon_{E}
$$

où $\mathbf{D}$ est l'opérateur de Hooke.

La résolution des différents problèmes associés à chaque sous-structure se fait indépendamment des autres sous-structures et peut ainsi être parallélisée.

Problème sur les interfaces

Le problème mécanique à résoudre sur chaque interface consiste déterminer $\underline{W}_{E}$ et $\underline{F}_{E}$ vérifiant la relation de comportement

$$
\mathrm{R}\left(\underline{W}_{E}, \underline{F}_{E}, \underline{W}_{E^{\prime}}, \underline{F}_{E^{\prime}}\right)=0
$$


où $\mathrm{R}$ est la relation (linéaire ou non) de comportement de l'interface $\Gamma_{E E^{\prime}}$ que nous expliciterons en détail en section 4.

Au bilan, on définit $\mathbf{s}=\sum_{E} \mathbf{s}_{E}$ comme étant la solution du problème sousstructuré telle que $\mathbf{s}_{E}=\left(\epsilon_{E}, \underline{W}_{E}, \sigma_{E}, \underline{F}_{E}\right)$ associé à chacune des sous-structures et ses interfaces voisines vérifie les équations [1] à [4].

\subsection{Aspect multi-échelle aux interfaces}

Contrairement à la plupart des approches multi-échelles, la séparation entre les échelles se fait ici au niveau des interfaces. Les quantités d'interface s'écrivent ainsi comme la somme d'une partie macro et d'une partie micro : $\underline{F}=\underline{F}^{M}+\underline{F}^{m}$ et $\underline{W}=\underline{W}^{M}+\underline{W}^{m}$. Les déplacements et efforts macro $\underline{W}^{M}$ et $\underline{F}^{\bar{M}}$ sont cherchés dans les espaces $\mathcal{W}_{E E^{\prime}}^{M}$ et $\mathcal{F}_{E E^{\prime}}^{M}$ définis plus bas. Les composantes macro sont obtenues par un projecteur $\Pi_{\Gamma_{E E^{\prime}}}$ vérifiant la relation de découplage des travaux micro et macro suivante :

$$
(\underline{F}, \underline{W})=\int_{\Gamma_{E E^{\prime}}} \underline{F} \cdot \underline{W} d \Gamma=\int_{\Gamma_{E E^{\prime}}} \underline{F}^{M} \cdot \underline{W}^{M} d \Gamma+\int_{\Gamma_{E E^{\prime}}} \underline{F}^{m} \cdot \underline{W}^{m} d \Gamma
$$

On a alors : $\underline{F}^{M}=\Pi_{\Gamma_{E E^{\prime}}}(\underline{F})$ et $\underline{F}^{m}=\left(i d-\Pi_{\Gamma_{E E^{\prime}}}\right)(\underline{F})$. Les composantes macro et micro du déplacement $\underline{W}$ s'écrivent de la même manière.

Afin d'expliciter l'opérateur de projection $\Pi_{\Gamma_{E E^{\prime}}}$ on note $\mathbf{e}_{E E^{\prime}}^{M}=\left(\underline{e}_{1}^{M}, . ., \underline{e}_{n_{M}}^{M}\right)$, une base de l'espace $\mathcal{F}_{E E^{\prime}}^{M}$. On a alors :

$$
\underline{F}^{M}=\Pi_{\Gamma_{E E^{\prime}}}(\underline{F})=\sum_{i=1}^{n_{M}}\left(\underline{F}, \underline{e}_{i}^{M}\right) \underline{e}_{i}^{M}=\sum_{i=1}^{n_{M}}\left[F^{M}\right]_{i} \underline{e}_{i}^{M}
$$

où $\left[F^{M}\right]_{i}$ représentent les composantes de $\underline{F}^{M}$ dans la base macroscopique $\mathbf{e}_{E E^{\prime}}^{M}$. On remarquera que la définition des composantes macro $\left[F^{M}\right]_{i=1 . . . n_{M}}$ est faite avant même toute discrétisation. Un choix classique consiste à utiliser un projecteur dit d'extraction de la «partie linéaire » des champs. La figure 3 illustre cette base de fonctions affines en dimension deux. Les composantes $\left[F^{M}\right]_{i}$ correspondent alors aux résultantes, moments et extensions de l'interface. La même base est utilisée pour $\mathcal{W}_{E E^{\prime}}^{M}$. Les composantes $\left[W^{M}\right]_{i}$ correspondent alors aux translations, rotations et allongement de l'interface.

Afin de transmettre l'information «globale » dans toute la structure $\Omega$, on impose aux efforts macro de vérifier systématiquement les conditions de transmission. L'espace associé est noté $\mathcal{F}_{a d}^{M}$.

$$
\mathcal{F}_{a d}^{M}=\left\{\underline{F}^{M} \mid \forall E, \forall E^{\prime} \in \mathbf{V}_{E}, \underline{F}_{E}^{M}+\underline{F}_{E^{\prime}}^{M}=0\right\}
$$

où $\mathbf{V}_{E}$ représente la liste des sous-structures voisines de $\Omega_{E}$. 


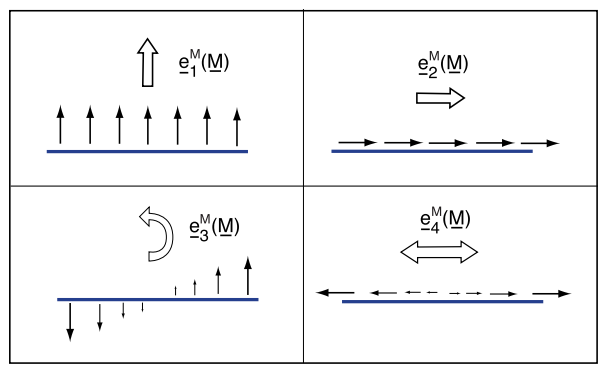

Figure 3. Base macroscopique linéaire $\left(n_{M}=4\right)$

\subsection{Stratégie itérative de résolution}

La solution $\mathbf{s}$ du problème est déterminée grâce l'algorithme itératif LATIN (Ladevèze, 1999). Contrairement à la plupart des méthodes « classiques » où pour avoir la réponse déplacement/effort il faut obligatoirement procéder par incrément et itérer à chaque pas de temps, la méthode LATIN donne à chaque itération une réponse admissible complète (sur tout l'intervalle de temps) et consiste à ajuster cette réponse jusqu'à obtenir la solution. La figure 4 illustre schématiquement le comportement d'une stratégie de résolution itérative incrémentale et celui de la méthode LATIN.

déplacement

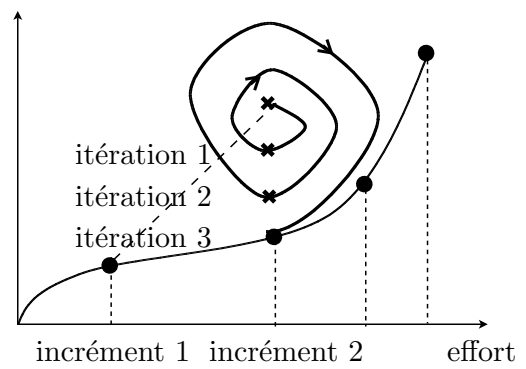

Méthode incrémentale

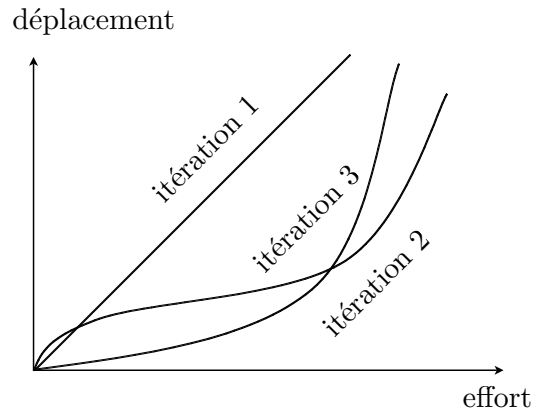

Méthode LATIN

Figure 4. Représentation schématique du comportement de deux stratégies de résolution

Le principe de base de la méthode LATIN est de séparer les difficultés en résolvant tour tour deux groupes d'équations $A_{d}$ et $\Gamma$ définis par : 


$$
\begin{aligned}
& A_{d} \quad \mid \begin{array}{l}
\text { - l'admissibilité statique de }\left(\sigma_{E}, \underline{F}_{E}\right) \\
- \text { l'admissibilité cinématique de }\left(\epsilon_{E}, \underline{W_{E}}\right) \\
- \\
\text { - la relation de comportement (ici linéaire) } \\
- \text { l'admissibilité de } \cup_{E}\left\{\underline{F}_{E}^{M}\right\} \in \mathcal{F}_{a d}^{M}
\end{array} \\
& \Gamma \quad \|- \text { le comportement des interfaces }
\end{aligned}
$$

$\Gamma$ regroupe les équations locales éventuellement non linéaires et $A_{d}$ les équations linéaires éventuellement globales.

La stratégie consiste chercher une solution qui vérifie alternativement les équations de $A_{d}$ puis celles de $\Gamma$ par le biais de deux directions de recherche, $E^{+}$et $E^{-}$(figure $5)$.

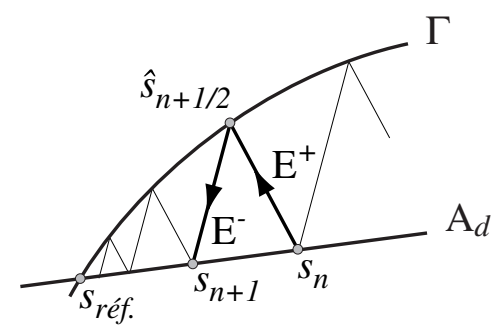

Figure 5. Schéma d'une itération de la méthode LATIN

A l'étape locale connaissant $\mathbf{s}_{n} \in A_{d}$, on cherche $\hat{\mathbf{s}}_{n+1 / 2} \in \Gamma$ vérifiant pour chaque interface $\Gamma_{E E^{\prime}}$ :

$$
\forall \underline{F}^{*} \in \mathcal{F}_{E E^{\prime}} \quad \int_{\Gamma_{E E^{\prime}}}\left(k^{-1}\left(\underline{\hat{F}}_{E}-\underline{F}_{E}\right)-\left(\underline{\hat{W}}_{E}-\underline{W}_{E}\right)\right) \cdot \underline{F}^{*} d \Gamma=0
$$

$k$ étant un scalaire positif. En pratique, $k=\kappa E / L$, où $E$ est le module d'Young, $L$ une dimension caractéristique de l'interface et $\kappa$ un scalaire multiplicateur. Dans le cas des interfaces parfaites, une étude a été menée pour déterminer une valeur optimale à $\kappa$ (Nouy, 2003). Dans les exemples traités ici, on prend $\kappa=0.5$.

A l'étape linéaire connaissant $\hat{\mathbf{s}}_{n+1 / 2} \in \Gamma$, on cherche $\mathbf{s}_{n+1} \in A_{d}$ qui vérifie pour chaque sous-structure :

$$
\forall \underline{F}^{*} \in \mathcal{F}^{m} \cup \mathcal{F}_{a d}^{M} \sum_{E} \int_{\partial \Omega_{E}}\left(k^{-1}\left(\underline{F}_{E}-\underline{\hat{F}}_{E}\right)+\left(\underline{W}_{E}-\underline{\hat{W}}_{E}\right)\right) \cdot \underline{F}_{E}^{*} d \Gamma=0 \text { [9] }
$$


Dans le cadre d'une résolution multi-échelle, la prise en compte de l'admissibilité macro : $\cup_{E}\left\{\underline{F}_{E}^{M}\right\} \in \mathcal{F}_{a d}^{M}$ modifie la direction de recherche $E^{-}$. L'équation [9] devient :

$$
\begin{aligned}
& \forall \underline{F}^{*} \in \mathcal{F} \quad \sum_{E} \int_{\partial \Omega_{E}}\left(k^{-1}\left(\underline{F}_{E}-\underline{\hat{F}}_{E}\right)+\left(\underline{W}_{E}-\underline{\hat{W}}_{E}\right)\right) \cdot \underline{F}_{E}^{*} d \Gamma= \\
& \sum_{E} \int_{\partial \Omega_{E}} \widetilde{W}_{E}^{M} \cdot \underline{F}_{E}^{*} d \Gamma
\end{aligned}
$$

où $\widetilde{W}_{E}^{M}$ est le multiplicateur de Lagrange correspondant à la contrainte de l'admissibilité des efforts macro. $\widetilde{W}_{E}^{M} \in \mathcal{W}_{a d, 0}^{M}$ l'espace des déplacements macro continus aux interfaces et nuls sur $\partial_{1} \Omega$ (Ladevèze et al., 2003).

$$
\forall \underline{\widetilde{W}}_{E}^{M *} \in \mathcal{W}_{a d, 0}^{M} \sum_{E} \int_{\partial \Omega_{E}} \widetilde{\widetilde{W}}_{E}^{M *} \cdot \underline{F}_{E} d \Gamma=\sum_{E} \int_{\partial \Omega_{E} \cap \partial_{2} \Omega} \widetilde{W}_{E}^{M *} \cdot \underline{F}_{d} d \Gamma
$$

La relation [11] exprime l'admissibilité des forces macro au sens faible. L'introduction des deux directions de recherche amène à un problème bien posé. Le problème peut être décomposé en deux parties : un problème micro défini sur chaque sous-structure et un problème macro défini sur l'ensemble des interfaces.

\section{Comportement des interfaces}

Le comportement d'une interface $\Gamma_{E E^{\prime}}$ entre deux sous-structures $\Omega_{E}$ et $\Omega_{E^{\prime}}$ dépend de la liaison que l'interface doit modéliser. Il peut être écrit comme une relation de comportement mixte entre les interdéplacements et les interefforts agissant sur l'interface. Nous donnons ici deux exemples de comportement d'interface.

L'interface parfaite

Les déplacements sont continus à travers l'interface et les efforts sont en équilibre. La relation de comportement se traduit alors par les deux équations : $\underline{F}_{E}+\underline{F}_{E^{\prime}}=0$ et $\underline{W}_{E}-\underline{W}_{E^{\prime}}=0$.

L'interface de contact avec frottement et jeu

Afin de respecter au mieux les conditions de frottement, il est nécessaire de prendre en compte leurs évolutions au cours du chargement (Champaney et al., 1997). Ainsi, le problème de frottement de Coulomb est discrétisé en temps. On note $\mu$ le coefficient de frottement de Coulomb, $\underline{n}_{E}$ désigne le vecteur normal $\Gamma_{E E^{\prime}}$ au point courant et sortant par rapport $\Omega_{E}$ et $j$ est le jeu initial (figure 6). $\mathbf{P}_{T}$ désigne le projecteur tangentiel associé à l'interface $\Gamma_{E E^{\prime}}$ tel que $\underline{W}_{E}=\left(\underline{n}_{E} \cdot \underline{W}_{E}\right) \underline{n}_{E}+\mathbf{P}_{T} \underline{W}_{E}$. 
REMARQUe. - Ici, l'hypothèse de petites perturbations étant établie, le vecteur normal $\underline{n}_{E}$ et le projecteur tangentiel $\mathbf{P}_{T}$ sont constants tout au long du calcul.

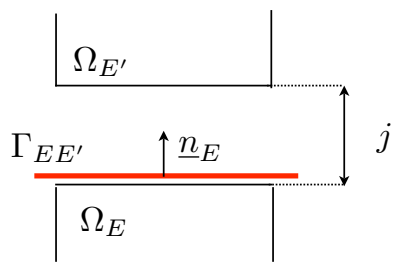

Figure 6. Notations pour une interface de contact

La relation de comportement se traduit alors au pas de temps $t$ par :

Contact unilatéral

- décollement

$$
\operatorname{Si} \underline{n}_{E} \cdot\left(\underline{W}_{E^{\prime}}^{t}-\underline{W}_{E}^{t}\right)+j>0 \text { alors } \underline{F}_{E}^{t}=\underline{F}_{E^{\prime}}^{t}=0
$$

- contact

$$
\operatorname{Si} \underline{n}_{E} \cdot\left(\underline{W}_{E^{\prime}}^{t}-\underline{W}_{E}^{t}\right)+j=0 \text { alors }\left\{\begin{array}{c}
\underline{F}_{E}^{t}+\underline{F}_{E}^{t}=0 \\
\underline{n}_{E} \cdot \underline{F}_{E}^{t}<0
\end{array}\right.
$$

Conditions de frottement

- adhérence

$$
\operatorname{Si}\left\|\mathbf{P}_{T} \underline{F}_{E}^{t}\right\|<\mu\left|\underline{n}_{E} \cdot \underline{F}_{E}^{t}\right| \text { alors } \mathbf{P}_{T}\left(\underline{V}_{E^{\prime}}^{t}-\underline{V}_{E}^{t}\right)=0
$$

- glissement

$$
\operatorname{Si}\left\|\mathbf{P}_{T} \underline{F}_{E}^{t}\right\|=\mu\left|\underline{n}_{E} \cdot \underline{F}_{E}^{t}\right| \text { alors }\left\{\begin{array}{c}
\mathbf{P}_{T}\left(\underline{V}_{E^{\prime}}^{t}-\underline{V}_{E}^{t}\right) \wedge \mathbf{P}_{T} \underline{F}_{E}^{t}=0 \\
\mathbf{P}_{T}\left(\underline{V}_{E^{\prime}}^{t}-\underline{V}_{E}^{t}\right) \cdot \mathbf{P}_{T} \underline{F}_{E}^{t} \geq 0
\end{array}\right.
$$

$\underline{V}_{E}^{t}$ étant la vitesse d'interface définie par :

$$
\underline{V}_{E}^{t}=\frac{W_{E}^{t}-\underline{W}_{E}^{t-1}}{\Delta t}=\frac{\Delta \underline{W}_{E}^{t}}{\Delta t}
$$

Dans le cas d'un problème quasi statique $\Delta t$ n'a pas de sens physique et la vitesse $\underline{V}_{E}^{t}$ est reliée à l'incrément de déplacement $\Delta \underline{W}_{E}^{t}$. 


\section{Résolution}

Dans cette section nous décrivons brièvement les différentes étapes de calcul effectuées au cours d'une itération LATIN.

\subsection{Résolution à l'étape linéaire : déterminer $\mathrm{s}_{n+1} \in A_{d}$}

\section{Problème micro}

Pour une sous-structure $\Omega_{E}$, la formulation faible en déplacement associée aux équations [1] à [3] et la vérification de la direction de recherche [10] conduit au problème micro suivant :

$$
\begin{aligned}
& \text { trouver }\left(\underline{u}_{E}, \underline{W}_{E}\right) \text { vérifiant : } \\
& \forall\left(\epsilon_{E}^{*}, \underline{W}^{*}\right) \text { admissible, } \quad \int_{\Omega_{E}} \operatorname{Tr}\left(\epsilon_{E}: \mathbf{D}: \epsilon_{E}^{*}\right) d \Omega+\int_{\partial \Omega_{E}} k \underline{W}_{E} \cdot \underline{W}^{*} d \Gamma= \\
& \int_{\partial \Omega_{E}}\left(\underline{\hat{F}}_{E}+k \underline{\hat{W}}_{E}+k \underline{\widetilde{W}}_{E}^{M}\right) \cdot \underline{W}^{*} d \Gamma
\end{aligned}
$$

Une discrétisation par éléments-finis standard est utilisée pour interpoler les champs de déplacements dans les sous-structures. L'interpolation des quantités d'interface est choisie judicieusement (Ladevèze et al., 2002) en considérant des élémentsfinis constants par morceaux sur les interfaces. En discrétisant l'équation [12] on obtient finalement :

$$
\left(\left[K_{E}\right]+\left[k_{E}\right]\right)\left[\underline{u}_{E}\right]=\left[\underline{\hat{F}}_{E, d}\right]+\left[k \underline{\widetilde{W}}_{E}^{M}\right]
$$

où $\left[K_{E}\right]$ est la matrice de rigidité éléments finis « classique » et $\left[k_{E}\right]$ est la matrice de rigidité de l'interface associée au terme $\int_{\partial \Omega_{E}} k \underline{W}_{E} \cdot \underline{W}^{*} d \Gamma$. $\left[\underline{\widehat{F}}_{E, d}\right]$ est le vecteur chargement connu correspondant au terme $\int_{\partial \Omega_{E}}\left(\underline{\hat{F}}_{E}+k \underline{\hat{W}}_{E}\right) \cdot \underline{W}^{*} d \Gamma \cdot \underline{\widetilde{W}}_{E}^{M}$ est la seule inconnue macro du problème [12]. Sa détermination passe par la résolution d'un problème macro sur l'ensemble des sous-structures.

\section{Problème macro}

Par linéarité du problème micro sur $\Omega_{E}$ et en utilisant [10], on peut définir $\mathbf{L}_{E}^{F}$ un opérateur homogénéisé liant les efforts macro aux multiplicateurs de Lagrange :

$$
\underline{F}_{E}^{M}=\mathbf{L}_{E}^{F}\left(\widetilde{\widetilde{W}}_{E}^{M}\right)+\underline{\hat{F}}_{E, d}^{M}
$$


où $\underline{\hat{F}}_{E, d}^{M}$ est le chargement macro « donné ». Le problème macro sur l'ensemble des sous-structures s'écrit alors :

trouver $\widetilde{W}_{E}^{M}$ tel que :

$$
\sum_{E} \int_{\partial \Omega_{E}} \widetilde{W}_{E}^{M *} \cdot\left(\mathbf{L}_{E}^{F}\left(\widetilde{W}_{E}^{M}\right)+\underline{\hat{F}}_{E, d}^{M}\right) d \Gamma=\sum_{E} \int_{\partial \Omega_{E} \cap \partial_{2} \Omega} \widetilde{W}_{E}^{M *} \cdot \underline{F}_{d} d \Gamma
$$

Après discrétisation on obtient :

$$
\left[\mathbf{L}^{F}\right]_{\mathbf{e}^{M}}\left[\widetilde{W}^{M}\right]_{\mathbf{e}^{M}}=\left[\underline{\widehat{F}}_{d}^{M}\right]_{\mathbf{e}^{M}}+\left[\underline{F}_{d}^{M}\right]_{\mathbf{e}^{M}}
$$

$[.]_{\mathbf{e}^{M}}$ désigne l'ensemble des composantes de la quantité considérée dans la base macro. Le vecteur $\left[\underline{\underline{F}}_{d}^{M}\right]_{\mathbf{e}^{M}}$ est associé au terme $\sum_{E} \int_{\partial \Omega_{E}} \widetilde{W}_{E}^{M *} \cdot \underline{\hat{F}}_{E, d}^{M} d \Gamma$ et le vecteur $\left[\underline{F}_{d}^{M}\right]_{\mathbf{e}^{M}}$ au terme $\sum_{E} \int_{\partial \Omega_{E} \cap \partial_{2} \Omega} \widetilde{W}_{E}^{M *} \cdot \underline{F}_{d} d \Gamma$. La taille du problème macro est donc de $n_{M} \times n_{i}$ où $n_{i}$ est le nombre d'interfaces et $n_{M}$ la dimension de la base macro de l'espace $\mathcal{F}_{E E^{\prime}}^{M}$ (figure 3).

\subsection{Résolution à l'étape locale : déterminer $\hat{\mathrm{s}}_{n+1 / 2} \in \Gamma$}

L'état de chaque point de l'interface de contact est donné de façon explicite grâce à deux indicateurs $g_{N}$ et $\underline{g}_{T}$ (Champaney et al., 1999; Ladevèze et al., 2002). Dans le cadre du contact avec frottement et en introduisant une discrétisation du chargement, ces deux quantités sont déterminées à partir de la solution au pas précédent.

L'indicateur de contact normal $g_{N}$ est défini par :

$$
g_{N}^{t+1}=\frac{1}{2} \underline{n}_{E} \cdot\left(\underline{V}_{E^{\prime}}^{t+1}-\underline{V}_{E}^{t+1}+\frac{\hat{W}_{E^{\prime}}^{t}-\underline{\hat{W}}_{E}^{t}}{\Delta t}\right)+\frac{j}{2}-\frac{1}{2 k} \underline{n}_{E} \cdot\left(\underline{F}_{E^{\prime}}^{t+1}-\underline{F}_{E}^{t+1}\right)
$$

L'indicateur de frottement $\underline{g}_{T}$ est défini par :

$$
\underline{g}_{T}^{t+1}=\frac{k}{2} \mathbf{P}_{T}\left(\underline{V}_{E^{\prime}}^{t+1}-\underline{V}_{E}^{t+1}\right)-\frac{1}{2} \mathbf{P}_{T}\left(\underline{F}_{E^{\prime}}^{t+1}-\underline{F}_{E}^{t+1}\right)
$$

Concernant le contact normal : $\mathrm{Si} g_{N}^{t+1}>0$ on a décollement, et si $g_{N}^{t+1} \leq 0$ on a contact des interfaces. D'autre part pour le contact tangentiel : $\operatorname{Si} \underline{g}_{T}^{t+1}<\mu\left|\underline{n}_{E} \cdot \underline{\hat{F}}_{E}^{t}\right|$ on a glissement et si $\underline{g}_{T}^{t+1} \geq \mu\left|\underline{n}_{E} \cdot \underline{\hat{F}}_{E}^{t}\right|$ on a adhérence des surfaces de contact. 
Suivant le cas de contact, on calcule finalement les composantes normales et tangentielles des quantités d'interface $\underline{\hat{W}}_{E}^{t+1}, \underline{\hat{W}}_{E^{\prime}}^{t+1}, \underline{\hat{F}}_{E}^{t+1}$ et $\underline{\hat{F}}_{E^{\prime}}^{t+1}$.

\subsection{Critère d'arrêt}

Le caractère non incrémental de la stratégie LATIN permet d'avoir très facilement accès à un indicateur de convergence. En effet, n'importe quel critère basé sur une mesure de la distance entre les solutions consécutives d'une même itération est un indicateur d'erreur pertinent (Ladevèze, 1999). Cet indicateur est calculé à la fin de chaque itération, et lorsque sa valeur passe en-dessous d'un critère $\epsilon_{l}$ l'algorithme est arrêté.

$$
\frac{\left\|\mathbf{s}_{n+1}-\hat{\mathbf{s}}_{n+1 / 2}\right\|}{\frac{1}{2}\left\|\mathbf{s}_{n+1}\right\|+\left\|\hat{\mathbf{s}}_{n+1 / 2}\right\|}<\epsilon_{l}
$$

En pratique on prend $\epsilon_{l}=10^{-5}$.

\subsection{Algorithme de résolution}

En bilan, la figure 7 donne l'algorithme général de résolution. Pour chaque étape, on note par entrée les quantités connues a priori et par sortie les quantités calculées.

\section{Description du détail structural}

La prise en compte de la forme et/ou de la position de détails structuraux est une étape importante dans le dimensionnement d'un système mécanique. L'aspect multiéchelle introduit permet de rendre compte du comportement global (échelle macro) de la structure tout en considérant de façon simple l'influence du détail structural (échelle micro). Ce travail constitue un autre genre d'étude paramétrique, entraînant généralement des temps de calcul prohibitifs. La description du détail géométrique au sein d'une sous-structure se fait ici indépendamment du maillage éléments finis selon la X-FEM. La technique des level set permet de modifier sa forme et/ou sa position au cours d'un calcul très simplement et à moindre coût.

\subsection{La méthode X-FEM}

Afin de s'affranchir des difficultés de maillage au niveau micro, une technique d'enrichissement locale comme la X-FEM est utilisée pour représenter le détail structural (Guidault et al., 2006). La séparation des échelles n'étant introduite qu'au niveau des interfaces, il est possible d'enrichir l'approximation du champ de déplacement $\underline{u}_{E}$ sur une sous-structure $E$ suivant la technique illustrée dans (Moës et al., 1999; Stolarska et al., 2001). Dans le cas d'un trou circulaire : 


\section{Initialisation}

Sur chaque sous-structure $\Omega_{E}$

- Calcul de $\left[K_{E}\right]$ et $\left[k_{E}\right]$

- Calcul de $\left[L_{E}^{F}\right]$

Sur chaque interface $\Gamma_{E E^{\prime}}$

- $\underline{\hat{W}}_{n=0}^{t=0}=0$

$\mathbf{s}_{n=0}^{t=1}=0 \quad \underline{V}_{n=0}^{t=1}=0$

Boucle LATIN $n=1 \cdots n^{\max }$

Boucle sur les pas de chargement $t=1 \cdots t^{\max }$

Etape locale

- Problème local sur les interfaces

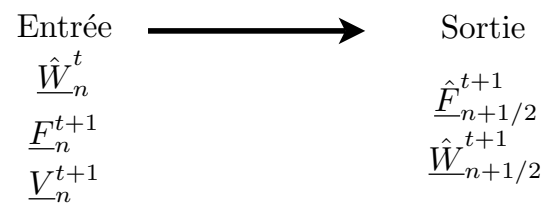

Etape linéaire

- Problème micro $\left(\left[K_{E}\right]+\left[k_{E}\right]\right)\left[\underline{\hat{u}}_{E}\right]=\left[\underline{\hat{F}}_{E, d}\right]$

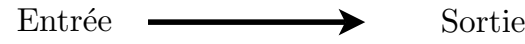

$\hat{\mathbf{s}}_{n+1 / 2}^{t+1} \quad \hat{\mathbf{s}}_{n+1}^{t+1}$

- Problème macro $\left[\mathbf{L}^{F}\right]_{\mathbf{e}^{M}}\left[\widetilde{\widetilde{W}}^{M}\right]_{\mathbf{e}^{M}}=\left[\underline{\hat{E}}_{d}^{M}\right]_{\mathbf{e}^{M}}+\left[\underline{F}_{d}^{M}\right]_{\mathbf{e}^{M}}$

Entrée Sortie

$\hat{\mathbf{s}}_{n+1}^{t+1} \longrightarrow \quad \underline{\widetilde{W}}_{n+1}^{M^{t+1}}$

- Problème micro $\left(\left[K_{E}\right]+\left[k_{E}\right]\right)\left[\underline{\widetilde{u}}_{E}\right]=\left[k \widetilde{\widetilde{W}}_{E}^{M}\right]$

Entrée $\longrightarrow$ Sortie

$\widetilde{W}_{n+1}^{M^{t+1}} \quad \widetilde{\mathbf{s}}_{n+1}^{t+1}$

- $\mathbf{s}_{n+1}^{t+1}=\hat{\mathbf{s}}_{n+1}^{t+1}+\widetilde{\mathbf{s}}_{n+1}^{t+1}$

Fin de boucle sur les pas de chargement

Test de convergence

\section{Fin boucle LATIN}

Figure 7. Algorithme de résolution 
- l'interpolation par la X-FEM du champ de déplacement dans la sous-structure $E$ est donnée par (Sukumar et al., 2000) :

$$
\underline{u}_{E_{h}}(\underline{x})=\sum_{i \in N} \varphi_{i}(\underline{x}) H(\underline{x}) \underline{u}_{i} \text { où } H(\underline{x})=\left\{\begin{array}{cc}
1 & \text { si } \underline{x} \text { est dans la matière } \\
0 & \text { si } \underline{x} \text { est dans le vide }
\end{array}\right.
$$

$N$ étant l'ensemble des nœuds du maillage, $\underline{u}_{i}$ les degrés de liberté correspondants et $H$ la fonction d'enrichissement. Seuls les éléments traversés par la frontière du trou font l'objet d'un traitement spécifique,

- l'intégration numérique de la formulation faible ne se fait pas sur la portion de l'élément située dans le vide. La figure $8 \mathrm{~b}$ représente les sous-éléments d'intégration considérés pour les éléments coupés par la frontière du trou. En particulier, on représente les points d'intégration dans la zone d'intérêt. Ce sous-découpage ne rajoute pas de degré de liberté au système,

- les nœuds à l'intérieur du trou dont aucun élément n'est coupé par sa frontière sont éliminés du maillage en supprimant leurs degrés de liberté dans le système final.

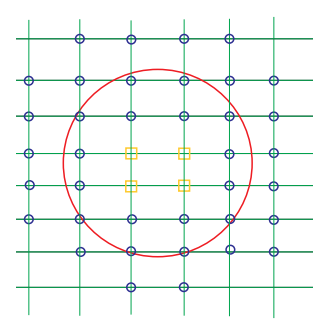

a)

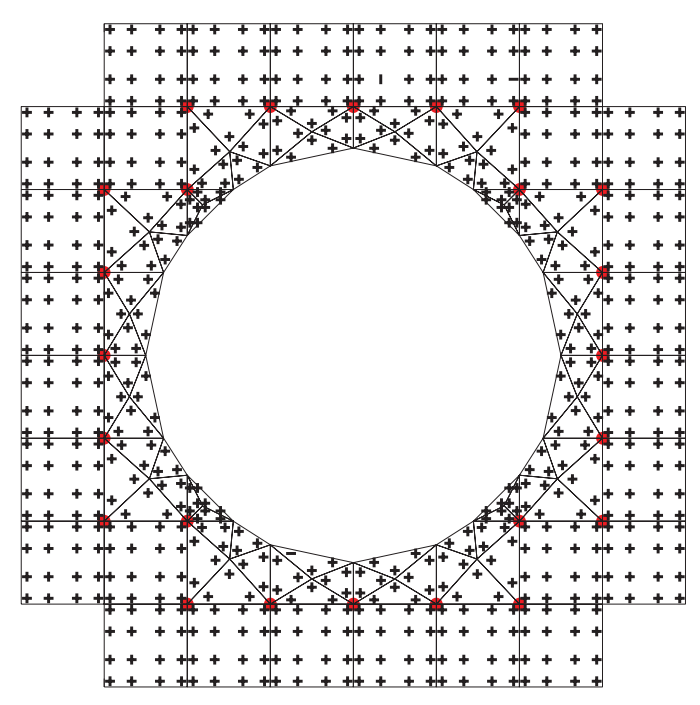

b)

Figure 8. a) Trou placé sur un maillage. Les næuds entourés d'un cercle sont assujettis à un traitement spécifique et les nœuds entourés d'un carré sont éliminés b) Représentation des points d'intégration à l'intérieur des éléments coupés par la frontière du trou 
REMARQUE. - L'enrichissement des nœuds d'une interface nécessite un traitement spécifique (Guidault et al., 2008). Ici le trou ne pourra pas chevaucher deux sousstructures.

\subsection{Modélisation du trou par level set}

La méthode des fonctions level set proposée par (Osher et al., 1988) est une technique numérique pour suivre l'évolution de détails structuraux facilement car indépendamment du maillage éléments finis. Dans le cas d'un trou, la fonction level set (fonction distance) est donnée par :

$$
\varphi(\underline{x})=\min \left(\left\|\underline{x}-\underline{x}_{c}\right\|-r_{c}\right)
$$

où $\underline{x}_{c}$ et $r_{c}$ sont respectivement la position de l'origine et le rayon du trou. Il est facile alors d'exprimer la fonction d'enrichissement $H$ en fonction de la fonction distance $\varphi$ comme suit :

$$
H(\underline{x})=\left\{\begin{array}{lll}
1 & \text { si } & \varphi(\underline{x}) \geq 0 \\
0 & \text { si } & \varphi(\underline{x})<0
\end{array}\right.
$$

\section{Stratégie multirésolution}

La méthode multirésolution présentée par (Boucard et al., 2003) permet d'économiser considérablement les temps de calcul pour des problèmes où un certain nombre de paramètres varient. Elle est basée sur le fait que l'algorithme LATIN peut être initialisé à partir de n'importe quelle solution vérifiant soit les conditions d'admissibilité dans les sous-structures soit les équations locales sur les interfaces. Dans le cas d'une étude paramétrique, pour un jeu de paramètres, on réinitialise la boucle LATIN avec la solution convergée correspondant à un autre jeu de paramètres. Lorsqu'un paramètre évolue légèrement, la solution globale du problème change peu, ainsi par la stratégie multirésolution, la convergence est atteinte plus rapidement en un nombre réduit d'itérations. Deux types de paramètres de conception seront étudiés dans la suite. Les coefficients de frottement $\mu$ caractéristiques des interfaces de contact et les paramètres géométriques d'un trou $p_{c}=\left(x_{c}, y_{c}, r_{c}\right)$, affectés aux sous-structures. La variation de ce dernier type de paramètre, engendre une perte de l'admissibilité de la solution s d'un calcul à un autre. Cela nécessite ainsi l'actualisation de la matrice de rigidité $\left[K_{E}\right]$ et de l'opérateur homogénéisé $\left[L_{E}^{F}\right]$ de la sous-structure concernée. Dans le cadre de cette étude seuls les quantités d'interface sont utilisées pour l'initialisation de l'algorithme LATIN. Ces champs vérifient dans tous les cas les équations locales de $\Gamma$, que la solution soit admissible ou non. Ainsi le calcul débute par une étape linéaire, connaissant $\left(\underline{\hat{W}}_{E}, \underline{\hat{F}}_{E}, \underline{\hat{W}}_{E^{\prime}}, \underline{\hat{F}}_{E^{\prime}}\right) \in \Gamma$ obtenus à la convergence du calcul précédent. 
Pour des problèmes généraux où les deux types de variables évoluent, la figure 9 représente le schéma de multirésolution considéré. Une boucle externe ( $\beta=$ $\left.1 \ldots \beta_{\max }\right)$ est effectuée sur les paramètres de détail géométrique $p_{c}$. Au sein d'une boucle interne $\left(\alpha=1 \ldots \alpha_{\max }\right)$ sur les paramètres de contact $\mu$, l'algorithme itératif pour $\mu^{(\alpha+1)}$ est initialisé avec les champs d'interfaces issus du calcul précédent, pour $\mu^{(\alpha)}$. Au début de chaque boucle interne $(\alpha=1)$ la solution convergée correspondante au paramètre $p_{c}^{(\beta)}$ est stockée. Ainsi, pour ce qui est de la boucle externe, l'algorithme LATIN pour $p_{c}^{(\beta+1)}$ est initialisé avec la solution du calcul pour $p_{c}^{(\beta)}$. Par conséquent, $\left[K_{E}\right]$ et $\left[L_{E}^{F}\right]$ sont recalculés uniquement dans la (les) sous-structure(s) d'intérêt et seulement $\beta_{\text {max }}$ fois.

Initialisation (figure 7)

Boucle sur les paramètres géométriques $p_{c}: \beta=1 \ldots \beta_{\max }$

- Mise à jour de la Level-Set

- Fonctions d'enrichissement $H$

- Actualisation de $\left[K_{E}\right]$ et $\left[L_{E}^{F}\right]$ dans la sous-structure percée

Boucle sur les paramètres de contact $\mu: \alpha=1 \ldots \alpha_{\max }$

- Initialisation LATIN avec les champs d'interfaces associés aux paramètres :

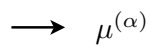

$\longrightarrow p_{c}^{(\beta)}$ si $\alpha=1$

- Boucle LATIN (figure 7) en multirésolution

Fin boucle sur $\mu$

Fin de boucle sur $p_{c}$

Figure 9. Etude paramétrique pour les deux types de variables de conception

\section{Exemples numériques}

\subsection{Pièce en contacts multiples}

Afin de valider la stratégie multi-échelle couplée à la multirésolution développée dans ce papier, reprenons l'exemple académique traité en monoéchelle par (Boucard et al., 2003) (figure 10a). On considère $\Omega_{1}, \Omega_{2}$ et $\Omega_{3}$ trois structures carrées identiques ( $h=50 \mathrm{~mm}$, module d'Young $E=2.10^{5} \mathrm{MPa}$, coefficient de Poisson $\nu=0,3$ ) en contact avec frottement. Dans un premier temps, on considère une seule sous-structure par domaine $\Omega_{i}, i=1,2,3$. Sur la figure $10 \mathrm{~b}$ les lignes en gras correspondent à 
la grille macro (interfaces) le maillage micro est représenté au sein de chaque sousstructure $(24 \times 24$ quadrangles bilinéaires $)$.

Cette étude paramétrique porte sur les coefficients de frottement $\mu_{1}$ et $\mu_{2}$ des deux interfaces de contact. Une pression verticale $P_{1}$, constituant la précharge, est tout d'abord appliquée sur $\Omega_{3}$, jusqu' sa valeur maximale de $50 M P a$. Puis, la structure centrale $\Omega_{2}$ est poussée contre une butée en appliquant le chargement horizontal progressivement de $030 \mathrm{MPa}$. Le jeu initial $j$ entre la butée et la structure est de $0.04 \mathrm{~mm}$. La figure 10b représente la déformée (micro) de l'assemblage ainsi que les déplacements (macro) des interfaces au dernier pas de temps dans le cas où le frottement est très important. Pour ce genre d'exemple assez simple, le problème macro représente déjà très bien le problème complet. La figure 11 représente l'évolution du déplacement horizontal du point $\mathrm{A}$ et celle de la composante verticale de la pression au point $\mathrm{B}$ au cours du chargement latéral $P_{2}$. Ces résultats sont obtenus pour les coefficients $\mu_{1}=0.1$ et $\mu_{2}=0.3$.

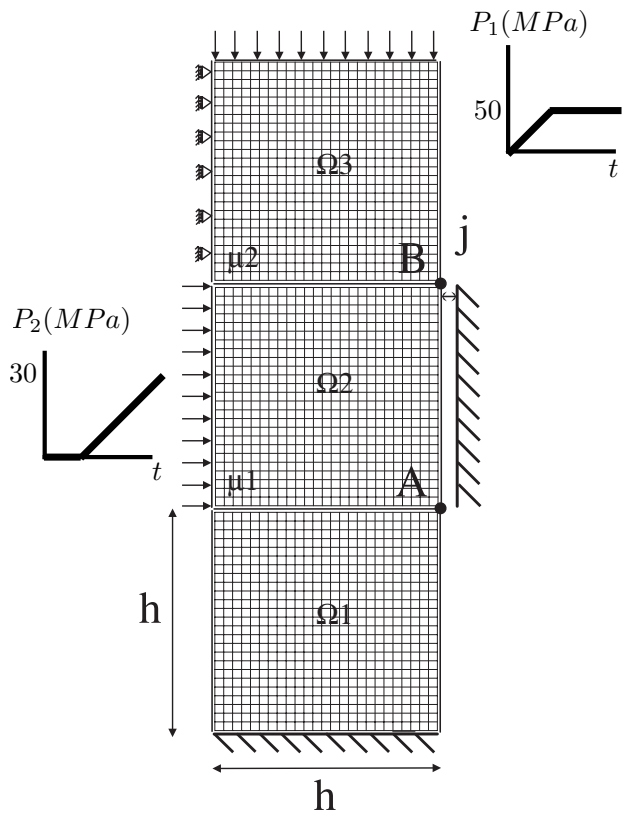

a)

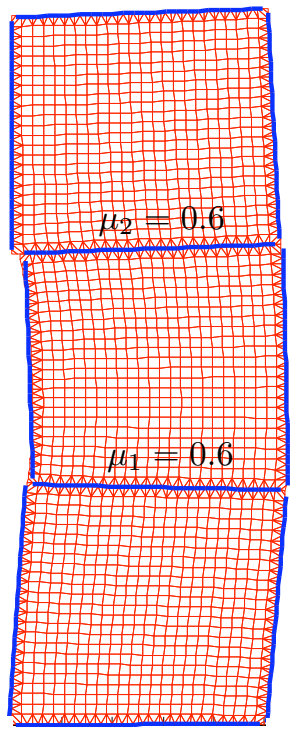

b)

Figure 10. a) Modèle b) déformée et déplacement macro pour $P_{2}=30 \mathrm{MPa}$

Pour l'analyse paramétrique à proprement parler, on effectue le calcul incrémental complet pour $13 \times 13$ couples de paramètres différents ( $\mu_{1}$ et $\mu_{2}$ varient de 00.6 par pas de 0.05). Pour chacun de ces couples, on relève la réaction horizontale appliquée 

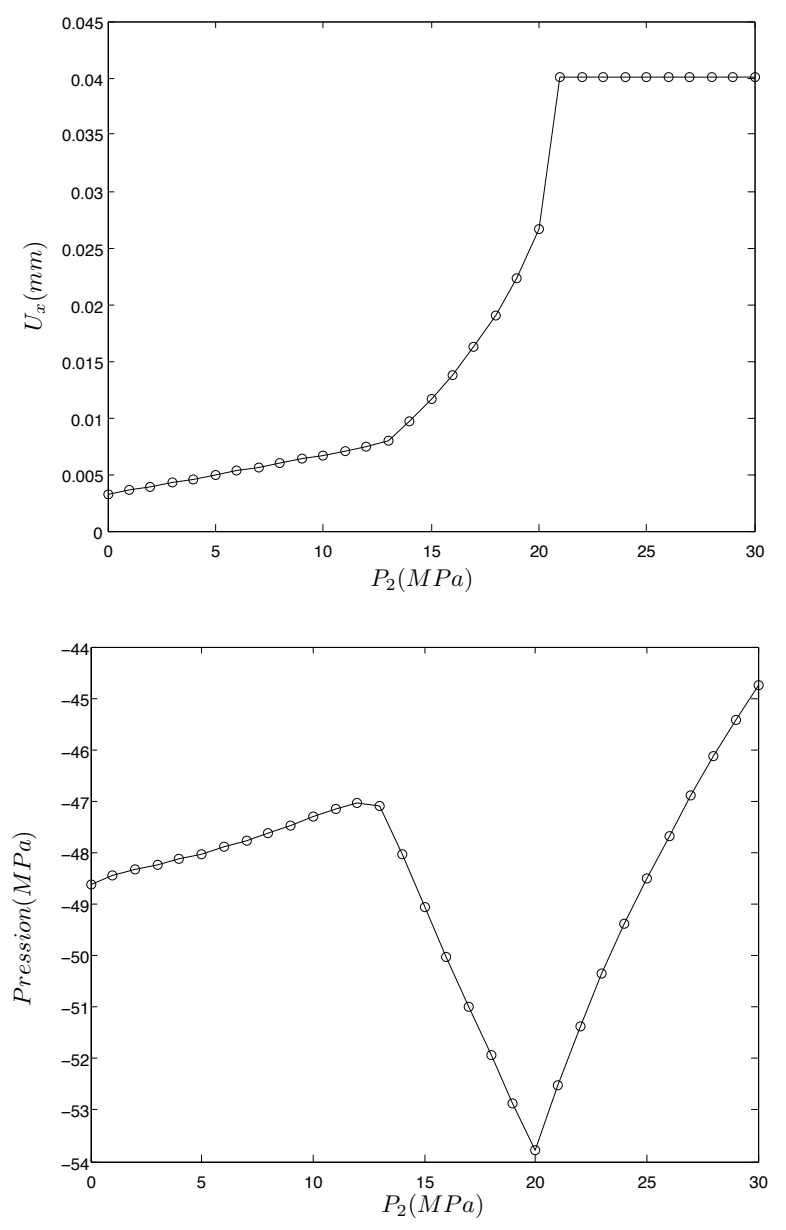

Figure 11. Déplacement horizontal du point A et pression verticale au point $B$

à la butée lorsque la totalité du chargement latéral est imposée $\left(F_{2}=1500 \mathrm{~N}\right)$, voir figure 12. Pour ce calcul $a$ priori très coûteux, la stratégie multirésolution est tout à fait adaptée. Par exemple, pour un coefficient $\mu_{1}$ fixé, le calcul pour $\mu_{2}=0.2$ sera initialisé partir de la solution convergée issue du calcul pour le coefficient $\mu_{2}=0.15$. Pour illustration, on extrait pour $\mu_{1}=0.3$ le nombre d'itérations ainsi que les temps CPU nécessaires la convergence (tableau 1). Le temps correspondant au premier calcul $\left(\mu_{2}=0\right)$ est pris comme référence et affecté de la valeur 1 unité de temps, toutes les valeurs sont ainsi normées par celui-ci. On effectue le même calcul en décomposant chaque domaine $\Omega_{i}$ en 4 sous-structures égales. Dans ces deux cas de partitionnement de domaine, on répertorie également le temps de calcul total ainsi que le temps requis si on n'avait pas utilisé la multirésolution. Pour évaluer ce dernier, on considère que 


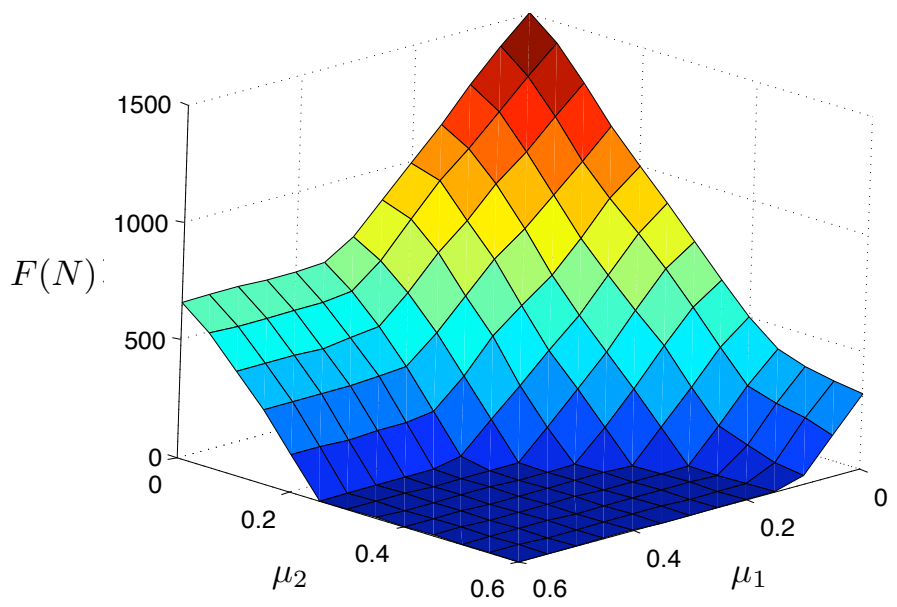

Figure 12. Composante horizontale de la résultante appliquée à la butée en fonction des coefficients de frottement

\begin{tabular}{|c|c|c||c|c|}
\hline$\mu_{1}=0.3$ & \multicolumn{2}{|c||}{ 1 sous-structure } & \multicolumn{2}{c|}{ 4 sous-structures } \\
\hline$\mu_{2}$ & Itérations & Temps & Itérations & Temps \\
\hline 0 & 25 & 1 & 33 & 1 \\
0.05 & 12 & 0.38 & 16 & 0.44 \\
0.1 & 11 & 0.34 & 14 & 0.39 \\
0.15 & 10 & 0.32 & 14 & 0.37 \\
0.2 & 9 & 0.29 & 13 & 0.34 \\
0.25 & 8 & 0.27 & 12 & 0.34 \\
0.3 & 7 & 0.22 & 10 & 0.28 \\
0.35 & 6 & 0.18 & 7 & 0.20 \\
0.4 & 4 & 0.13 & 5 & 0.14 \\
0.45 & 4 & 0.12 & 4 & 0.11 \\
0.5 & 3 & 0.10 & 4 & 0.11 \\
0.55 & 3 & 0.10 & 3 & 0.09 \\
0.6 & 3 & 0.10 & 4 & 0.11 \\
\hline \hline Temps total & 3.55 & \multicolumn{3}{c|}{3.92} \\
Ratio & 3.56 & 3.28 \\
\hline
\end{tabular}

Tableau 1. Gain apporté par la stratégie multirésolution lors de la variation d'un paramètre de conception 
le temps nécessaire pour chaque variation de paramètre est identique au temps du premier calcul, c'est à dire à $13 \times 1=13$. Finalement, on donne pour information le ratio temps sans multirésolution/temps avec multirésolution. Ce rapport montre sans équivoque la force de la méthode multi-échelle/multirésolution. Pour le calcul complet (lorsque les deux coefficients varient simultanément) le ratio est de 4.4.

REMARQue. - Les résultats obtenus dans cette étude (figures 11 et 12) sont comparables à ceux donnés par (Boucard et al., 2003) dans la version monoéchelle de la méthode. Par contre, le gain apporté par la multirésolution en multi-échelle en terme de temps de calcul n'est pas significatif par rapport à l'approche monoéchelle et ceci pour les deux types de sous-structuration. Sur un cas aussi simple que celui-ci, il n'est pas étonnant de ne pas surpasser le mono-échelle. Cet exemple académique permet néanmoins de valider notre méthode.

\subsection{Plaque trouée en traction}

Le but de ce second exemple est de valider la méthode X-FEM couplée à la stratégie multirésolution. Pour cela nous prenons un exemple tiré de (Clément et al., 2007), nos résultats sont comparés à des calculs éléments finis « classiques » effectués sous CAST3M (Verpeaux et al., 1988) où le trou est maillé explicitement. On considère une plaque carrée trouée ( $h=3 \mathrm{~mm}, E=1 \mathrm{MPa}, \nu=0)$ en traction simple $(\sigma=0.1$ $M P a$ ), voir figure 13. Dans cette étude, les trois paramètres sont les coordonnées $x_{c}$, $y_{c}$ et le rayon $r_{c}$ du trou. La plaque est constituée d'une seule sous-structure.

Cette étude est effectuée avec la stratégie multirésolution. Les conditions aux limites étant prises via les interfaces un nombre d'itérations minimal est nécessaire à la convergence, ici le premier calcul (pour le premier jeu de paramètre) requiert 6 itérations et $30 s$ de temps CPU pour converger. Les calculs suivants quant à eux convergent en 1 seule itération et en $20 \mathrm{~s}$. Le gain en terme de nombre d'itérations montre la performance de la stratégie dans le cas de la variation de paramètres géométriques avec la méthode X-FEM. Par contre, si le gain en terme de temps de calcul n'est pas très bon ici, ce n'est pas à cause de la stratégie multirésolution elle même, mais parce qu'à chaque calcul on doit actualiser la matrice de rigidité et l'opérateur homogénéisé de toute la plaque, ce qui est une étape toujours coûteuse dans un code de calcul éléments finis. La figure 13 représente le déplacement vertical du point $\mathrm{A}$ en fonction de la position du trou pour deux rayons différents. La corrélation parfaite des surfaces de réponse permet de valider la méthode d'enrichissement local pour la description d'un trou introduite ici.

\subsection{Pièce trouée en contacts multiples}

Reprenons l'exemple 7.1 et considérons que le paramètre de l'étude est le rayon et la position d'un trou percé dans la structure centrale, les coefficients de frotte- 

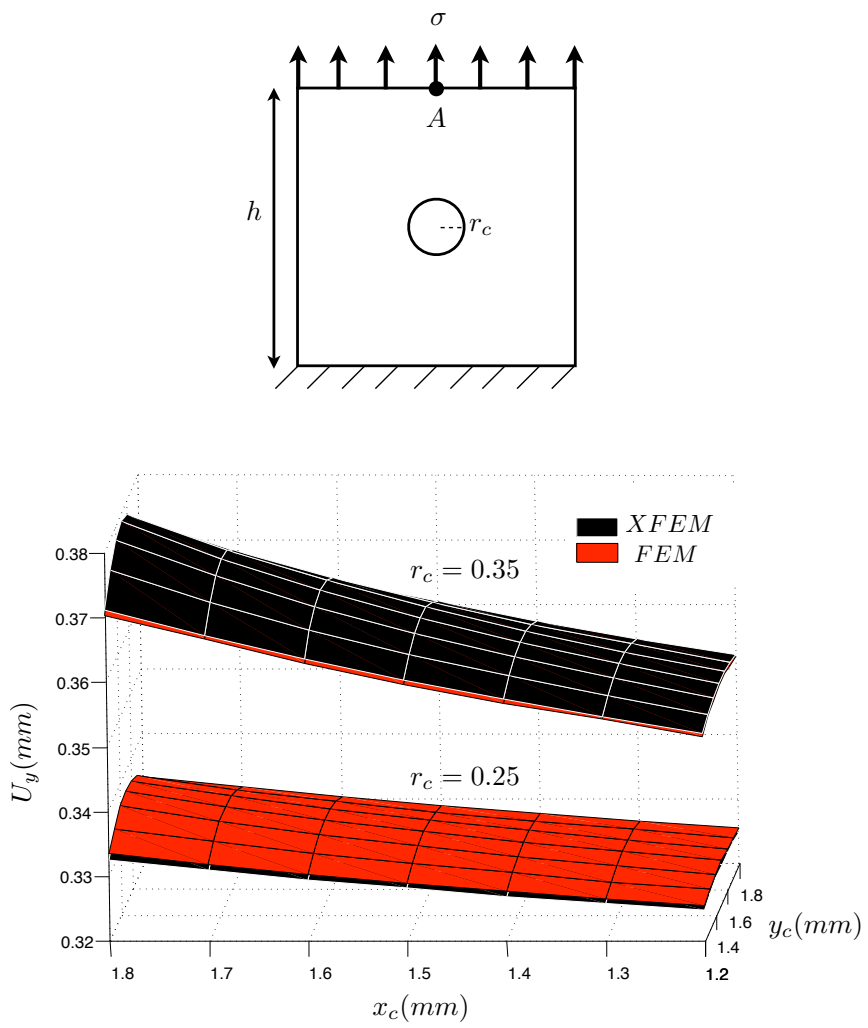

Figure 13. Traction simple d'une plaque trouée. Déplacement du point A en fonction de la position du trou

ment étant dans un premier temps fixés $\mu_{1}=0.35$ et $\mu_{2}=0.25$. Les domaines $\Omega_{i}$ sont décomposés en 9 sous-structures. La figure 14 représente la décomposition de la structure $\Omega_{2}$, le trou pouvant évoluer dans la sous-structure centrale. Afin d'avoir une description fine dans la zone du détail géométrique, le maillage est pris trois fois plus dense dans la sous-structure centrale. Les maillages sont dits incompatibles et le raccord au niveau des interfaces parfaites se fait à efforts micro nuls (Guidault et al., 2007). La relation de comportement se traduit par les équations :

$$
\left\{\begin{array}{l}
W_{E}^{M}-\underline{W}_{E^{\prime}}^{M}=0 \\
\underline{F}_{E}^{M}+\underline{F}_{E^{\prime}}^{M}=0
\end{array} \quad \text { et } \quad \underline{F}_{E}^{m}=\underline{F}_{E^{\prime}}^{m}=0\right.
$$


Ce type de raccord correspond à un raccord en « moyenne » de déplacement, c'està-dire qu'à partir de la base linéaire choisie dans cette étude. Par dualité, l'interface transmet uniquement la partie linéaire des efforts.

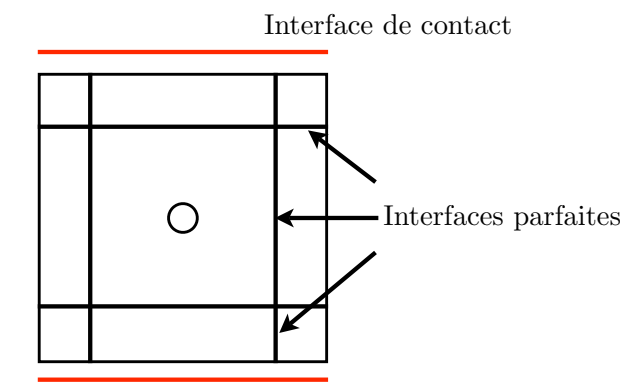

Figure 14. Partitionnement en neuf sous-structures de la structure percée $\Omega_{2}$

Le trou est au centre de la structure $\left(x_{c}=25 \mathrm{~mm}\right.$ et $\left.y_{c}=75 \mathrm{~mm}\right)$ et on étudie l'influence de la taille de son rayon sur la composante horizontale $F$ de la résultante appliquée à la butée. Cette composante est égale à $14 N$ en absence de trou (figure 12). En outre, on compare ces résultats, sur la figure 15, d'une part avec ceux donnés par l'approche des éléments finis classiques et d'autre part avec ceux obtenus lorsque l'on ne considère qu'une seule sous-structure par pièce de l'assemblage. L'augmentation de la taille du trou implique un décollement progressif des surfaces de contact, conduisant à un meilleur glissement de la structure $\Omega_{2}$ donc à une résultante de plus en plus importante (de 15 à $24 \mathrm{~N}$ ). Les résultats sont relativement semblables pour les trois approches considérées.

\begin{tabular}{|cc|ccccc|}
\hline$r_{c}=3 \mathrm{~mm}$ & \multicolumn{5}{|c|}{$x_{c}(\mathrm{~mm})$} \\
& & 23 & 24 & 25 & 26 & 27 \\
\hline \multirow{3}{*}{$y_{c}(\mathrm{~mm})$} & 73 & 1 & 0.18 & 019 & 0.22 & 0.24 \\
& 74 & 0.17 & 0.17 & 0.18 & 0.2 & 0.21 \\
& 75 & 0.19 & 0.17 & 0.18 & 0.19 & 0.20 \\
& 76 & 0.22 & 0.17 & 0.18 & 0.20 & 0.20 \\
& 77 & 0.23 & 0.17 & 0.19 & 0.21 & 0.22 \\
\hline
\end{tabular}

Tableau 2. Gain apporté par la stratégie multirésolution lors de la variation de la position du trou (deux paramètres de conception).

La figure 16 représente la déformée (amplifiée d'un facteur 300) de la structure pour le dernier pas de chargement $\left(P_{2}=30 \mathrm{MPa}\right)$ et pour deux rayons différents. Dans la suite de l'étude on considérera uniquement la décomposition en 9 sousstructures avec maillage incompatible. Le rayon du trou étant maintenant fixé, on 

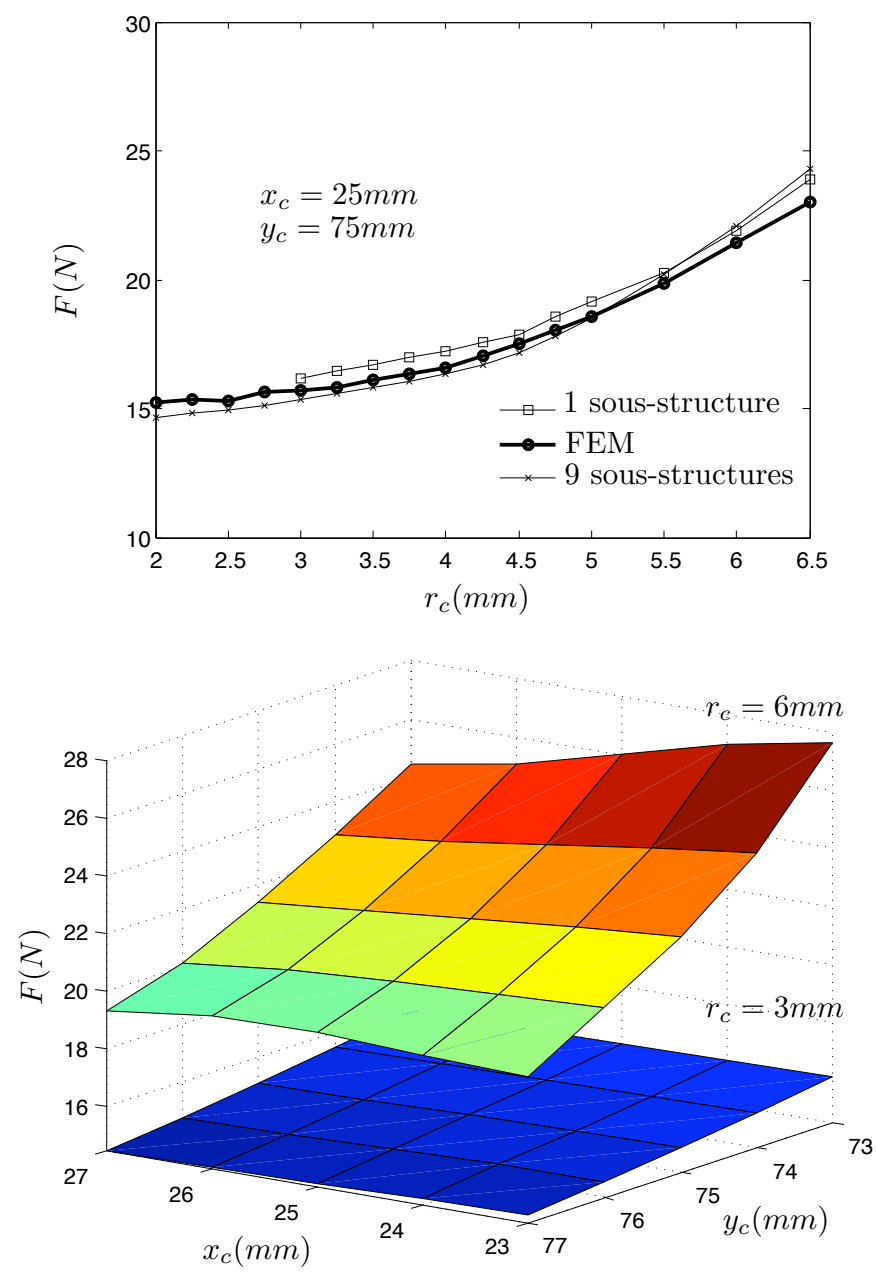

Figure 15. Influence du trou sur la réponse de la structure : $\mu_{1}=0.35$ et $\mu_{2}=0.25$

cherche à étudier l'influence de la position du trou sur cette même résultante (figure 15). Le tableau 2 donne les temps CPU (normés par le premier calcul) nécessaires à l'obtention de tous les points de la surface de réponse correspondant au rayon $r_{c}=3$ $\mathrm{mm}$. Le ratio temps sans multirésolution/temps avec multirésolution de 4.4 montre de nouveau l'efficacité de la stratégie multirésolution dans le cas de la variation de paramètres géométriques.

REMARQUE. - La possibilité d'introduire une interface incompatible à efforts micro nuls permet d'avoir un maillage de la finesse requise uniquement dans la zone 

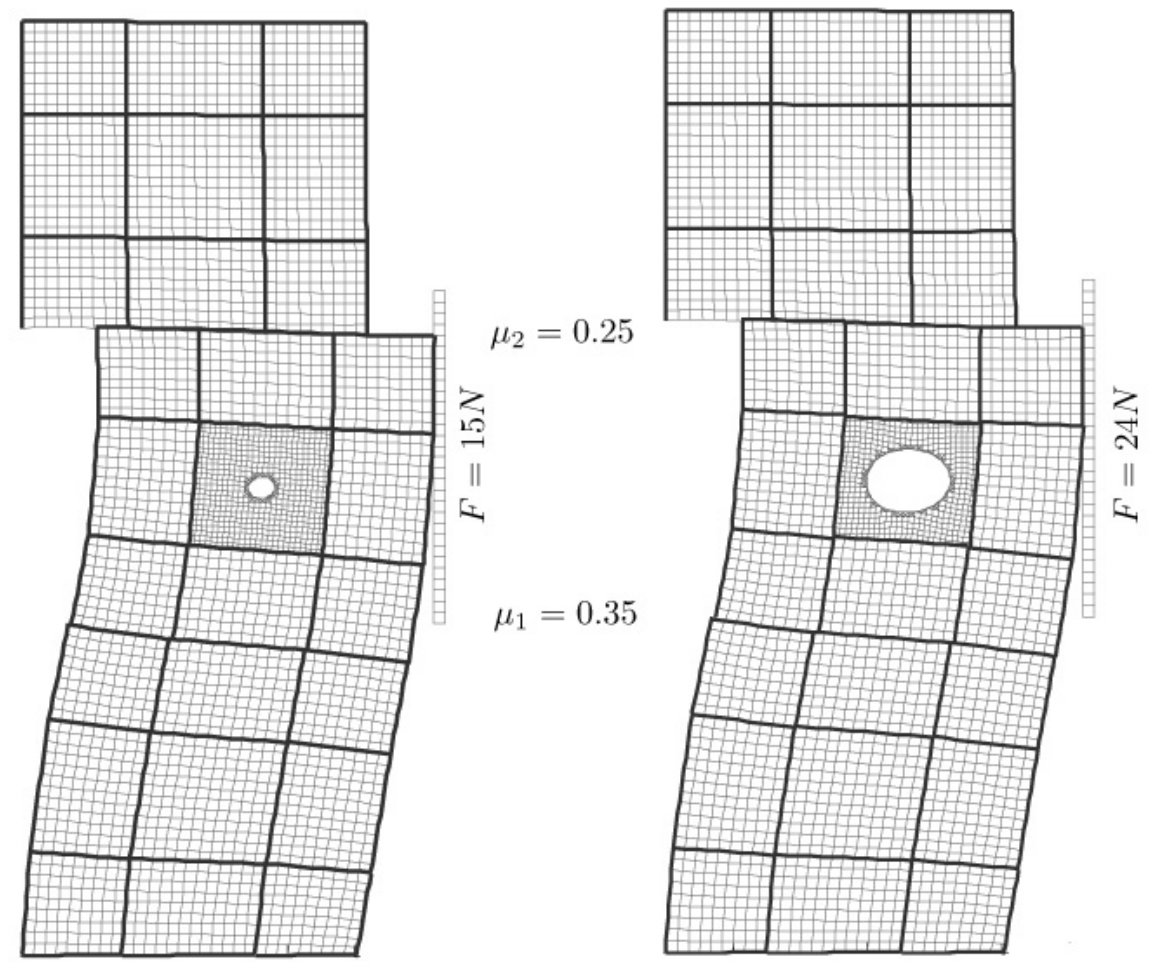

Figure 16. Déformée de la structure pour deux diamètres de trou

d'intérêt sans avoir aucune contrainte de maillages « micro » entre la sous-structure raffinée et les autres sous-structures. Les rigidités et opérateurs homogénéisés de la sous-structure raffinée sont calculés indépendamment sans « répercussion » sur les sous-structures voisines.

Finalement, ce même problème est traité en faisant varier le maximum de variables de conception. Considérons les cinq paramètres en simultané : les deux coefficients de frottement $\mu_{1}, \mu_{2}$ et les trois variables géométriques caractérisant le trou $x_{c}, y_{c}$ et $r_{c}$. La stratégie multirésolution se base sur le schéma de la figure 9 . Ici $13 \times 13 \times 5 \times 5 \times 5=$ 21125 calculs sont effectués et le gain en termes de temps CPU apporté est de 5.2, montrant de nouveau la performance de la stratégie multirésolution.

En bilan, au vu des différentes études paramétriques effectuées, dans le cas 2D, le gain lié à la stratégie multirésolution, bien que légèrement croissant avec le nombre de calcul, est globalement constant quel que soit le nombre de paramètres de conception 
considéré. Dans le cas des problèmes 3D on peut s'attendre à des gains beaucoup plus importants comme cela a été constaté en mono-échelle (Boucard et al., 2003).

\section{Conclusion}

La stratégie mise en place au service d'études paramétriques de détails structuraux au sein de structures à multiples contacts combine plusieurs méthodes performantes. La séparation des échelles de la stratégie micro-macro consiste à définir un problème macro de taille réduite sur les interfaces dans le but d'accélérer la convergence de l'algorithme itératif. L'approche X-FEM permet de décrire les détails structuraux indépendamment du maillage et la stratégie multirésolution réduit considérablement les temps de calcul. L'intégration du contact frottant comme comportement d'interface nécessite une résolution quasi statique, gérée de façon intrinsèque par l'algorithme LATIN. Les exemples numériques 2D considérés dans cette étude valident d'une part la méthode proposée et montrent, d'autre part, l'économie en terme d'itérations et de temps de calcul faite grâce à l'introduction de la stratégie multirésolution. Ce gain sera d'autant plus significatif dans des problèmes 3D. A terme, il sera envisageable de considérer des détails de géométrie plus complexe.

\section{Remerciements}

Ces travaux ont reçu le soutien de l'Agence nationale pour la recherche dans le cadre du programme RNTL 2005 : projet Optimisation multidisciplinaire (OMD).

\section{Bibliographie}

Boucard P., Champaney L., « A suitable computational strategy for the parametric analysis of problems with multiple contact », International Journal for Numerical Methods in Engineering, vol. 57, p. 1259-1281, 2003.

Boucard P., Champaney L., « Approche multirésolution pour l'étude paramétrique d'assemblages par contact et frottement », Revue européenne des éléments finis, vol. 13, p. 437-448, 2004.

Champaney L., Cognard J., Dureisseix D., Ladevèze P., « Large scale applications on parallel computers of a mixed domain decomposition method », Computational Mechanics, vol. 19, p. 253-263, 1997.

Champaney L., Cognard J., Ladevèze P., « Modular analysis of assemblages of threedimensional structures with unilateral contact conditions », Computers and Structures, vol. 73, p. 249-266, 1999.

Clément A., Nouy A., Schoefs F., Moës N., « Méthode des éléments finis stochastiques étendus pour le calcul de structures à géométrie aléatoire », $8^{e}$ colloque national en calcul des structures, Giens, France, 2007.

Combescure A., Rannou J., Gravouil A., « A multigrid eXtended Finite Element Method for elastic crack growth simulation », European Journal of computational Mechanics, vol. 16, p. 161-182, 2007. 
1036 Revue européenne de mécanique numérique. Volume 16 - n 8/2007

Daux C., Moës N., Dolbow J., Sukumar N., Belytschko T., « Arbitrary branched and intersecting cracks with the extended finite element method », International Journal for Numerical Methods in Engineering, vol. 48, p. 1741-1760, 2000.

Fish J., Yuan Z., « Multiscale enrichment based on partition of unity », International Journal for Numerical Methods in Engineering, vol. 62, p. 1341-1359, 2005.

Guidault P., Allix O., Champaney L., Cornuault C., « Une approche micro-macro pour le suivi de fissure avec enrichissement local », Revue européenne de mécanique numérique, vol. 15, p. 187-198, 2006.

Guidault P., Allix O., Champaney L., Cornuault C., « A multiscale Extended Finite Element Method for crack propagation », Computer Methods in Applied Mechanics and Engineering, vol. 197, p. 381-399, 2008.

Guidault P., Allix O., Champaney L., Navarro J., « A two-scale approach with homogenization for the computation of cracked structures », Computers and Structures, vol. 85, p. 13601371, 2007.

Kim Y., Yoon G., « Multi-resolution multi-scale topology optimization : a new paradigm », International Journal of Solids and Structures, vol. 37, p. 5529-5559, 2000.

Ladevèze P., Loiseau O., Dureisseix D., « A micro-macro and parallel computational strategy for highly heterogeneous structures », International Journal for Numerical Methods in Engineering, vol. 52, n¹-2, p. 121-138, 2001.

Ladevèze P., Nonlinear Computational Structural Mechanics - New Approaches and nonIncremental Methods of Calculation, Springer Verlag, 1999.

Ladevèze P., Nouy A., « On a multiscale computational strategy with time and space homogenization for structural mechanics », Computer Methods in Applied Mechanics and Engineering, vol. 192, p. 3061-3088, 2003.

Ladevèze P., Nouy A., Loiseau O., « A multiscale computational approach for contact problems », Computer Methods in Applied Mechanics and Engineering, vol. 191, p. 4869-4891, 2002.

Moës N., Dolbow J., Belytschko T., « A finite element method for crack growth without remeshing », International Journal for Numerical Methods in Engineering, vol. 46, p. 131-150, 1999.

Nouy A., Une stratégie de calcul multiéchelle avec homogénéisation en temps et en espace pour le calcul de structures fortement hétérogènes, Thèse de doctorat, ENS Cachan, 2003.

Osher S., Sethian J., « Fronts propaging with curvature-dependent speed : Algorithms based on Hamilton-Jacobi formulations », Journal of Computational Physics, vol. 79, p. 12-49, 1988.

Stolarska M., Chopp D. L., Moës N., Belytschko T., « Modelling Crack Growth by Level Sets and the Extended Finite Element Method », International Journal for Numerical Methods in Engineering, vol. 51, n 8 8. p. 943-960, 2001.

Sukumar N., Chopp D. L., Moës N., Belytschko T., « Modeling holes and inclusions by level sets in the extended finite-element method », Computer Methods in Applied Mechanics and Engineering, vol. 190, p. 6183-6200, 2000.

Verpeaux P., Charras T., Millard A., Une approche moderne du calcul des structures, Fouet JM, Ladevèze P, Ohayon R editors. Calcul des Structures et Intelligence artificielle, 1988. 